\title{
Summing Large-N Towers in Colour Flow Evolution
}

\section{Simon Plätzer*}

Theory Group, DESY, Hamburg

E-mail: simon.plaetzer@desy.de

\begin{abstract}
We present an algorithm to systematically approximate multiple soft gluon exchange in the colour flow basis, based on investigating the colour structure of the (one-loop) soft anomalous dimension matrix and a successive exponentiation of classes of large- $N$ contributions. We anticipate that the method presented in this contribution is well-suited to deal with many external legs by means of Monte Carlo methods and will form the basis of further improving parton shower algorithms towards next-to-leading logarithmic accuracy.
\end{abstract}

Loops and Legs in Quantum Field Theory - LL 2014,

27 April - 2 May 2014

Weimar, Germany

\footnotetext{
* Speaker.
} 


\section{Introduction}

In order to reliably interpret current and upcoming measurements at the LHC, precise QCD predictions for multi-jet final states are indispensable. Not only at the level of fixed-order precision calculations, but as well by means of combining such predictions with analytic resummation and/or parton shower event generators such as to sum leading perturbative contributions to all orders in perturbation theory. Fixed-order calculations at leading and next-to-leading order in the strong coupling are by now standard and available in an automated way, and a similar level has been reached to analytically resum a large class of observables [1]. Combining NLO QCD corrections with event generators [2-7] is a well established area of research, the combination of analytic resummation and event generators has recently been pioneered, as well [8].

The efficient treatment of QCD colour structures is central to both fixed-order and resummed perturbation theory, eventually leading to an improvement of parton shower event generators beyond the leading- $N$ approximation and hence towards next-to-leading logarithmic (NLL) or even higher accuracy. In this contribution we consider the colour flow basis, which has led to tremendously efficient implementations of tree-level amplitudes [9-11], impacting both leading order calculations, as well as one-loop corrections, see e.g. [12]. This colour basis is closely linked to determining initial conditions for parton showering, but has so far received little attention in the context of analytic resummation. We will consider soft gluon evolution in the colour flow basis. While for a fixed, small number of partons the exponentiation of the soft gluon anomalous dimension matrix can be performed exactly, the general case for a large number of legs is rapidly becoming intractable. This limitation thus prevents insight into the soft gluon dynamics of highmultiplicity systems as relevant to both improved parton shower algorithms $[13,14]$ as well as colour reconnection models $[15,16]$.

By tracking the flow of colour charge, the colour flow basis organizes QCD amplitudes in a physically very transparent way. We stress that this basis is not tied to the large- $N$ limit. The underlying construction principle is to trade off the familiar components of the gluon field with respect to the adjoint representation, $A_{a}^{\mu}$, for components with respect to the fundamental and antifundamental representations, $\mathscr{A}^{\mu i}{ }_{\bar{i}}=A_{a}^{\mu}\left(t^{a}\right)^{i}{ }_{\bar{i}}$. This can be done at the level of the QCD Lagrangian and a full set of Feynman rules is, for example, given in [9]. Within the colour flow basis we consider a standard decomposition of the amplitude into colour structures ${ }^{1}|\sigma\rangle$ labeled by permutations indicating the connections between fundamental and anti-fundamental indices,

$$
\left|\mathscr{M}_{n}\right\rangle=\sum_{\sigma} \mathscr{M}_{n, \sigma}|\sigma\rangle, \quad|\sigma\rangle=\left|\begin{array}{ccc}
1 & \ldots & m \\
\sigma(1) & \ldots & \sigma(m)
\end{array}\right\rangle=\delta_{i_{\overline{\sigma(1)}}^{i_{1}}} \ldots \delta_{i_{\overline{\sigma(m)}}^{i_{m}}}^{i} .
$$

A pictorial representation of basis tensors for two colour flows is given in figure 1.

Multiple soft gluon exchanges can be resummed by evolving the hard process amplitude with the (in general path-ordered) $)^{2}$ exponential of the soft anomalous dimension matrix $\Gamma$,

$$
\left|\mathscr{M}_{n}^{\prime}\right\rangle=e^{\Gamma}\left|\mathscr{M}_{n}\right\rangle, \quad \Gamma=\sum_{\alpha \neq \beta} \Gamma^{\alpha \beta} \mathbf{T}_{\alpha} \cdot \mathbf{T}_{\beta} .
$$

\footnotetext{
${ }^{1}$ The reader is referred to [17] for details on the notation

${ }^{2}$ We here consider the case that path ordering is not required; it is straightforward to keep track of path-ordering effects in the most general case.
} 


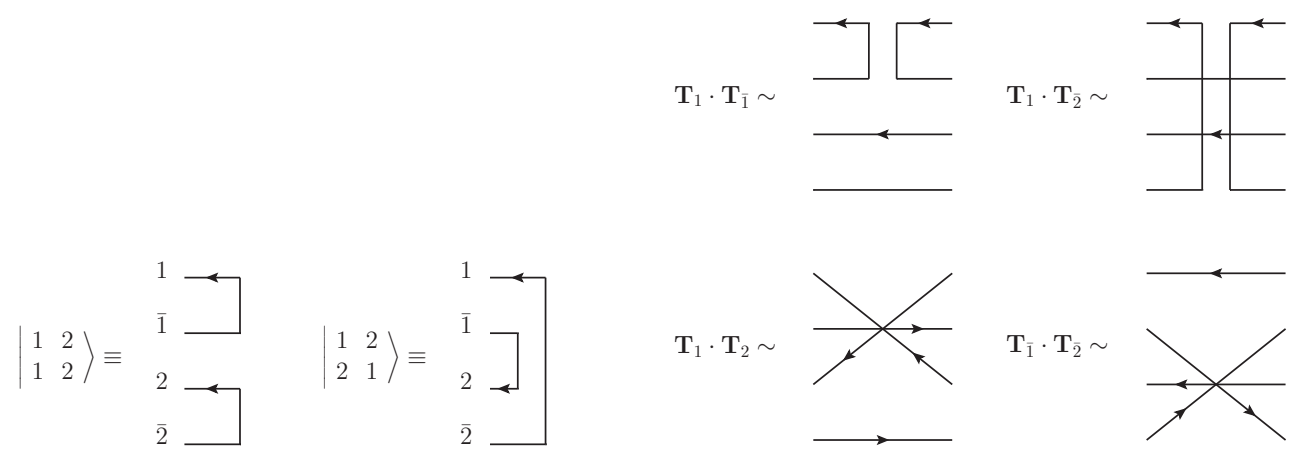

Figure 1: Left: Example of colour flow basis tensors; each line corresponds to a Kronecker symbol linking fundamental and anti-fundamental representation indices. Right: Non-diagonal contributions to the soft anomalous dimension matrix; see text for details.

\section{Soft Anomalous Dimensions in the Colour Flow Basis}

The strategy chosen in the present work is now to translate the colour charge correlators $\mathbf{T}_{\alpha} \cdot \mathbf{T}_{\beta}$ with respect to external leg indices $\alpha, \beta$ into the associated (anti-)fundamental indices $i, j, \bar{i}, \bar{j}$, and then expressing these using the Fierz identity of $\mathrm{SU}(N)$, e.g.

$$
\mathbf{T}_{i} \cdot \mathbf{T}_{j}=\frac{1}{2}\left(\delta_{j}^{i^{\prime}} \delta_{i}^{j^{\prime}}-\frac{1}{N} \delta_{i}^{i^{\prime}} \delta_{j}^{j^{\prime}}\right), \quad \mathbf{T}_{i} \cdot \mathbf{T}_{\bar{j}}=-\frac{1}{2}\left(\delta_{\bar{j}^{\prime}}^{i^{\prime}} \delta_{i}^{\bar{j}}-\frac{1}{N} \delta_{i}^{i^{\prime}} \delta_{\bar{j}^{\prime}}^{\bar{j}}\right)
$$

We then find a very simple structure for matrix elements of the soft anomalous dimension for an arbitrary number of external legs,

$$
[\tau|\Gamma| \sigma]=\left(-N \Gamma_{\sigma}+\frac{1}{N} \rho\right) \delta_{\tau \sigma}+\Sigma_{\tau \sigma}
$$

where $\Gamma_{\sigma}, \rho$ and $\Sigma_{\tau \sigma}$ are linear combinations of the original kinematic coefficients $\Gamma^{\alpha \beta}$. Owing to the structure of soft gluon exchange at one-loop, $\Sigma_{\tau \sigma}$ is non-vanishing only if the permutations $\sigma$ and $\tau$ differ by exactly one transposition. $\Gamma_{\sigma}$ originates from the first, non-diagonal, terms in the Fierz identity for which the basis vector considered is an eigenvector; $\rho$ originates from the $1 / \mathrm{N}$, diagonal terms, whilst $\Sigma_{\tau \sigma}$ contains the action of the non-diagonal terms truly mapping one colour flow into a distinct one. Examples of these non-diagonal, and genuinely large- $N$ suppressed 'colour reconnectors' are given in figure 1.

\section{Summation of Large- $N$ Towers}

The structure of the soft anomalous dimension matrix directly suggests to treat $\Sigma_{\tau \sigma}$ as a perturbation to the trivially exponentiable contributions $\Gamma_{\sigma}$ and $\rho$. More precisely, we perform a resummation of diagonal terms which can be expected to be of order one, and successively add 
powers of the non-diagonal contributions, schematically:

$$
\begin{aligned}
\text { at LC }: & 1+\gamma N+\gamma^{2} N^{2}+\ldots \\
\text { at NLC }: & \left(\gamma+\frac{\gamma}{N}\right)\left(1+\gamma N+\gamma^{2} N^{2}+\ldots\right) \\
\text { at NNLC }: & \left(\gamma+\frac{\gamma}{N}\right)^{2}\left(1+\gamma N+\gamma^{2} N^{2}+\ldots\right),
\end{aligned}
$$

where $\gamma$ refers to a kinematic coefficient. The trivial contribution $\rho$ can be dealt with in two ways: Either we consider this a perturbation as well (since it is suppressed by $1 / N^{2}$ relative to the leading part), this being the case for the series pattern sketched above, or we absorb it into a redefinition of $\Gamma_{\sigma}$. We will refer to 'primed' resummation in the latter case.

The structure of the successive approximations can readily be inferred from casting the matrix exponential into a serious in powers of $1 / N$,

$$
\left[\tau\left|e^{\Gamma}\right| \sigma\right]=\sum_{l=0}^{\infty} \frac{(-1)^{l}}{N^{l}} \times \sum_{k=0}^{l} \frac{(-\rho)^{k}}{k !} \sum_{\sigma_{0}, \ldots, \sigma_{l-k}} \delta_{\tau \sigma_{0}} \delta_{\sigma_{l-k} \sigma}\left(\prod_{\alpha=0}^{l-k-1} \Sigma_{\sigma_{\alpha} \sigma_{\alpha+1}}\right) \times R\left(\left\{\sigma_{0}, \ldots, \sigma_{l-k}\right\},\left\{\Gamma_{\sigma}\right\}\right),
$$

where

$$
R(\sigma, \Gamma)=\left[\prod_{\alpha=0}^{\# \text { uniq }(\sigma)-1} \frac{1}{d_{\alpha}(\sigma) !} \frac{\partial^{d_{\alpha}(\sigma)}}{\partial^{d_{\alpha}(\sigma)} \Gamma_{\sigma_{\alpha}}}\right] \sum_{\alpha=0}^{\# \text { uniq }(\sigma)-1} e^{-N \Gamma_{\sigma_{\alpha}}} \prod_{\beta=0, \beta \neq \alpha}^{\# \text { uniq }(\sigma)-1} \frac{\left(\Gamma_{\sigma_{\beta}} / \Gamma_{\sigma_{\alpha}}\right)^{d_{\beta}(\sigma)}}{\Gamma_{\sigma_{\alpha}}-\Gamma_{\sigma_{\beta}}} .
$$

$\mathrm{N}^{d} \mathrm{LC}$ approximations are then obtained by truncating the sum over $l$ at the $d$ 'th order. Notice that at $\mathrm{N}^{d} \mathrm{LC}$, instead of the full matrix exponentiation ${ }^{3}$, only $d-1$ matrix multiplications are required. Indeed, since $\Sigma_{\tau \sigma}$ turns out to be extremely sparse, while a simple recipe is available to calculate its non-vanishing elements, a matrix multiplication in the usual sense is not even required and we propose to use a Monte Carlo sum over the possible colour structures to implement these expressions in a practical application.

As a simple example, consider NLC, where we find

$$
\left[\tau\left|e^{\Gamma}\right| \sigma\right]=\delta_{\tau \sigma} e^{-N \Gamma_{\sigma}}\left(1+\frac{\rho}{N}\right)-\frac{1}{N} \Sigma_{\tau \sigma} \frac{e^{-N \Gamma_{\tau}}-e^{-N \Gamma_{\sigma}}}{\Gamma_{\tau}-\Gamma_{\sigma}}+\text { NNLC }
$$

Notice that this approximation is in principle sufficient for a (NLL) resummation to be matched to a NLO calculation, since upon fixed-order expansion we have

$$
\left.\left[\tau\left|e^{\Gamma}\right| \sigma\right]\right|_{\mathrm{NLC}}=\delta_{\tau \sigma}+[\tau|\Gamma| \sigma]+\mathscr{O}\left(\gamma^{2}\right)
$$

reproducing the singularity structure present in the one-loop calculation.

\section{Higher Orders and Numerics}

The evaluation of the successive approximations can easily be casted into an algorithmic prescription which, along with all other required bookkeeping and infrastructure, have been implemented in the $\mathrm{C}++$ library CVolver, to be published in the near future. CVolver features transparent interfaces to implement anomalous dimensions and hard amplitudes, and provides all the

\footnotetext{
${ }^{3}$ We remind the reader that the dimensionality of these matrices grows a factorial in the number of external legs.
} 

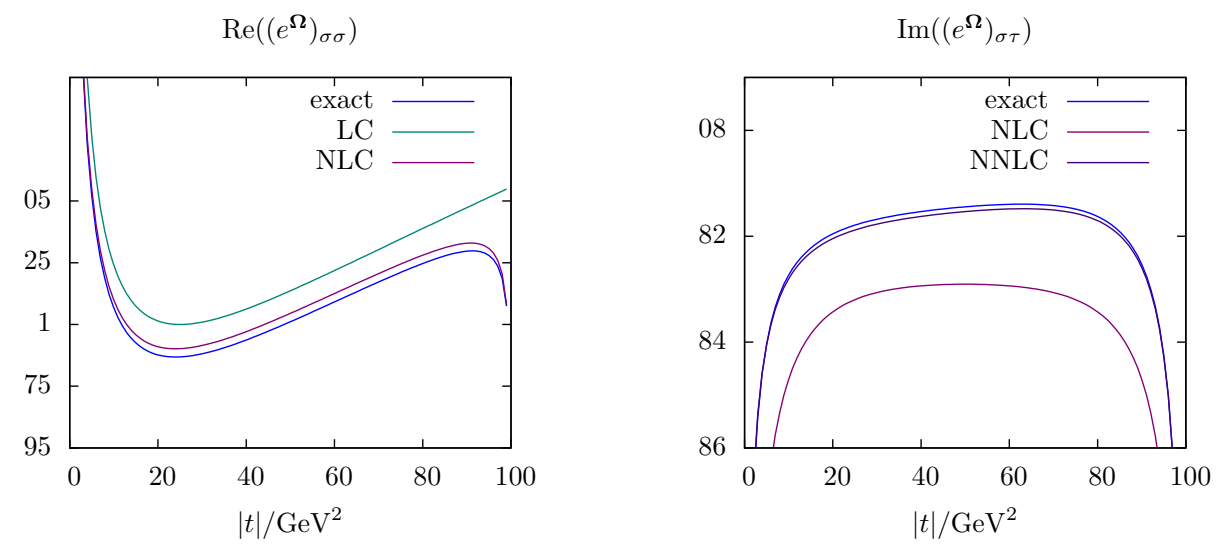

Figure 2: Numerical evaluation of evolution matrix elements for a jet veto setting as functions of the momentum transfer in the hard process. Left: real part of a diagonal element; right: imaginary part of an off-diagonal element.

utilities for performing Monte Carlo sums over colour flows. Within the present work we have used the program to numerically assess the convergence pattern of the successive approximations in a case where the full evolution can still be solved exactly. We directly investigate matrix elements of the matrix exponential, noting that for a large number of legs a sufficiently high order in the large- $N$ approximation is required to get non-vanishing estimates for all matrix elements. The impact of these contributions, describing transitions between colour flows which differ by a large number of permutations ultimately needs to be investigated at the level of squared amplitudes. As the colour basis chosen is not orthogonal, we have

$$
|\mathscr{M}|^{2}=\langle\mathscr{M} \mid \mathscr{M}\rangle=\mathscr{M}_{\sigma}^{\dagger} S_{\sigma \tau} \mathscr{M}_{\tau},
$$

with

$$
S_{\sigma \tau}=\langle\sigma \mid \tau\rangle=N^{m-\# \text { transpositions }(\sigma, \tau)} .
$$

One could be tempted to include the leading- $N$ pattern of the squared amplitudes along with the approximations performed in the soft gluon evolution. We argue not to perform such an approximation to assess the convergence of the evolved amplitude subject to a certain large- $N$ approximation, since the two expansions are entirely unrelated: the summation of large- $N$ towers discussed before is used to approximate the matrix exponential, while a large- $N$ approximation within the squared amplitude would also imply to approximately treat the hard input process, which ultimately could obscure the convergence pattern of the soft gluon evolution.

As a definite example, we consider the setting of a jet veto in hadronic dijet production; the evolution of quark scattering can easily be calculated exactly and will serve as a cross check for $\mathrm{N}^{d} \mathrm{LC}$ approximations. We find that at $\mathrm{N}^{3} \mathrm{LC}$, the approximation agrees with the exact result at sub-permille level, where primed resummations typically require one order less to reach the same level of accuracy. Examples of matrix elements as functions of the momentum transfer are given in figure 2 . 


\section{Outlook}

The work presented here can serve as a basis for investigating various aspects connected to soft gluon evolution of processes with a large number of external legs, amongst them:

- Exact soft gluon evolution, when full exponentiation is feasible, to be combined with colour flow based matrix element generators;

- Approximate soft gluon evolution upon checking successive approximations for their convergence and truncating at a reliable order;

- Improvement of parton shower algorithms to reach NLL accuracy at a certain colour approximation; and

- A better understanding of colour reconnection models by explicitly providing perturbative input to the purely phenomenological reasoning considered so far.

Computationally, the transparent and algorithmic structure of soft gluon evolution in the colour flow basis makes it possible to avoid any explicit linear algebra operations by performing the required matrix multiplications within Monte Carlo sums over colour flows, leading to a very efficient implementation.

\section{Acknowledgments}

I would like to thank the organizers for this very interesting and stimulating workshop. My work has been supported in part by the Helmholtz Alliance 'Physics at the Terascale'.

\section{References}

[1] A. Banfi, G. P. Salam, and G. Zanderighi, Phys.Lett. B584, 298 (2004), hep-ph/0304148.

[2] S. Frixione and B. R. Webber, JHEP 06, 029 (2002), hep-ph/0204244.

[3] P. Nason, JHEP 11, 040 (2004), hep-ph/0409146.

[4] Z. Nagy and D. E. Soper, JHEP 10, 024 (2005), hep-ph/0503053.

[5] S. Platzer and S. Gieseke, Eur.Phys.J. C72, 2187 (2012), 1109.6256.

[6] S. Hoeche, F. Krauss, M. Schonherr, and F. Siegert, JHEP 1209, 049 (2012), 1111.1220.

[7] L. Hartgring, E. Laenen, and P. Skands, JHEP 1310, 127 (2013), 1303.4974.

[8] S. Alioli et al., (2012), 1211.7049.

[9] F. Maltoni, K. Paul, T. Stelzer, and S. Willenbrock, Phys.Rev. D67, 014026 (2003), hep-ph/0209271.

[10] C. Duhr, S. Hoeche, and F. Maltoni, JHEP 0608, 062 (2006), hep-ph/0607057.

[11] T. Gleisberg and S. Hoeche, JHEP 0812, 039 (2008), 0808.3674.

[12] C. Reuschle and S. Weinzierl, (2013), 1310.0413.

[13] A. Schofield and M. H. Seymour, JHEP 1201, 078 (2012), 1103.4811. 
[14] S. Platzer and M. Sjodahl, JHEP 1207, 042 (2012), 1201.0260.

[15] M. Sandhoff and P. Z. Skands, (2005).

[16] S. Gieseke, C. Rohr, and A. Siodmok, Eur.Phys.J. C72, 2225 (2012), 1206.0041.

[17] S. Platzer, Eur.Phys.J. C74, 2907 (2014), 1312.2448. 